\title{
Stratification of the level of aerobic fitness based on heart rate variability parameters in adult males at rest
}

\author{
Wollner Materko ${ }^{*}$
}

ORIGINAL ARTICLE

\begin{abstract}
This study aimed to determine a model for stratifying the maximum oxygen uptake $\left(\mathrm{VO}_{2} \mathrm{max}\right)$ based on heart rate variability (HRV) parameters at rest. It was applied 70 young adult physically active male. After recording the resting tachogram with a heart rate monitor for $5 \mathrm{~min}$, a maximal incremental test was performed to measure $\mathrm{VO}_{2}$ max. Based on $\mathrm{VO}_{2}$ max percentile, participants were divided into two groups of 35: high (HG) and low (LG) aerobic fitness group. Groups were compared according to: (1) $\mathrm{VO}_{2} \mathrm{max}$; (2) Classical HRV parameters and the cardiac deceleration rate (CDR). HG presented significant higher $\mathrm{VO}_{2}$ max than LG $\left(48.61 \pm 6.70\right.$ vs $\left.34.62 \pm 3.81 \mathrm{ml} \cdot \mathrm{kg}^{-1} \cdot \mathrm{min}^{-1}\right)$. The HG higher values in the time domain HRV parameters, as well as in the high frequency contents in the frequency domain. Also, CDR index was significant higher for HG than LG $(\mathrm{p}<0.01)$. The model for predicting $\mathrm{VO}_{2}$ max was obtained by backward stepwise multivariate logistic regression assuming as independent variables CDR and pNN50. This model presented $85.74 \%$ positive predictive value, $88.51 \%$ negative predictive value and $87.16 \%$ total accuracy. In conclusion, the use of the proposed model was able to stratify the level of aerobic fitness in young healthy adult males at rest.

Keywords: heart rate variability, cardiac deceleration rate, aerobic fitness, power spectral density
\end{abstract}

\section{INTRODUCTION}

The evaluation of aerobic fitness is usually measured through the assessment of maximum oxygen consumption $\left(\mathrm{VO}_{2} \max \right)$, as a measure of the oxygen transported in the blood and pumped by the heart to the working muscles and efficiency of the muscles to use that oxygen. Therefore, increasing the aerobic fitness leads to an increasing ability of the heart to supply oxygen and energy to human body (Wasserman \& Whipp, 1975). This is often estimated at maximal cardiopulmonary exercise testing (CPET). However, the high cost of the equipment can make it impractical (Mezzani et al., 2009).

The analysis of heart rate variability (HRV) has been used in the clinic as a prognostic tool to assess the autonomic control of the heart rate fluctuations (Task Force, 1996). Most of the studies on healthy populations showed the effects of physical training and HRV indices (Kawaguchi et al., 2007; Marocolo, Nadal \& Barbosa, 2007; Melanson \& Freedson, 2001;
Rosenwinkel et al., 2001). However, other studies showed the opposite (Grant et al., 2013; Bosquet et al., 2007). Thus, the present study investigated another possibility of indices, based on HRV analysis, since it is already associated with physical fitness (Nasario-Junior et al., 2014; Nasario-Junior et al., 2015a). In this sense, the acceleration and deceleration indices of the RR interval series were studied (Bauer et al., 2006).

Recently, Bauer et al. (2009) introduced the phase-rectification signal averaged (PRSA) approach to HRV analysis, that consists in separately assessing the accelerating and the decelerating phases of $\mathrm{R}-\mathrm{R}$ interval series, in order to estimate the sympathetic and the parasympathetic contributions to heart rate control. Particularly the decelerating capacity index is being useful to predict mortality after myocardial infarction (Bauer et al., 2009), to estimate physical conditioning (Nasario-Junior et al., 2014; Nasario-Junior et al., 2015a) and autonomic control status in Chagas disease

\footnotetext{
Manuscript received at May 25 ${ }^{\text {th } 2017 ; ~ A c c e p t e d ~ a t ~ O c t o b e r 30 ~} 2017$

${ }^{1}$ Laboratory of Human Movement Biodynamic, School of Physical Education, Federal University of Amapá, Macapá, Brazil.

*Corresponding author: Address: Rod. Juscelino Kubitschek km 2, Jardim Marco Zero, Macapá city, AP. Zip Code: 68.903-419 E-mail: wollner.materko@unifap.br
} 
patients (Nasario-Junior et al., 2015b). On the other hand, it is still unknown how these parameters relate with $\mathrm{VO}_{2} \max$.

A simple, safe and accurate procedure for estimating the $\mathrm{VO}_{2} \max$ would benefit the stratification of aerobic fitness, only based on the resting parameters derived from HRV and on the phase-rectification signal averaged through the development of a mathematical model and without the need for a maximum cardiopulmonary test. Therefore, the purpose of the present study was to stratify the degree of aerobic fitness using a logistic model based on parameters derived from HRV and PRSA.

\section{METHODS}

\section{Participants}

Participated in the present study 70 voluntary male, 19-29 years old, non-smokers, with no history of cardiopulmonary disease. All participants were physical education students with different levels of aerobic fitness, and none was taking any medication. The participants were divided into two groups of 35 subjects, according to the percentile $50^{\text {th }}$ of the $\mathrm{VO}_{2} \max$ distribution to physical conditioning assessment: high aerobic fitness group (HG) and low aerobic fitness group (LG). The Ethics Committee of the Institution of Universidade Federal do Rio de Janeiro (CAAE: 12207013.5.0000.5257), and an informed written consent was obtained from all participants. The study was conducted according to the instructions of the Helsinki Declaration of 2008.

\section{Anthropometric Measurements}

Testing procedures and time commitment required for participation in this study were verbally explained to potential participants in a familiarization session. Anthropometric data were collected by the same experienced evaluator, who assessed for height, body mass and skinfold measurement. Body mass was measured to the nearest $0.1 \mathrm{~kg}$ and the height was measured in centimetres using a mechanical scale with stadiometer (Filizola, Brazil). Body fat was estimated by skinfold thickness measurements. A skinfold calliper (Cescorf,
Brazil) was used to take skinfolds measurements to the nearest $0.1 \mathrm{~mm}$ on the right side of the body. Body density was estimated based on skinfolds (Jackson \& Pollock, 1978) and the percent body fat was determined based on body density (Siri, 1961).

\section{Experimental procedures}

All the tests were conducted in a quiet room with temperature maintained at $22^{\circ} \mathrm{C}$. All volunteers were instructed to avoid physical exercise, alcoholic beverages and tobacco products in the preceding 24 hours, as well as not consume a heavy meal for, at least, three hours prior to testing and to remain hydrated throughout the tests.

In the first visit to the lab, all participants were instructed to lie in supine position for 5 min at rest while breathing normally. A heart rate monitor Polar RS810 (Polar, Finland), working at a sampling rate of $1000 \mathrm{~Hz}$ was used to record R-R intervals (RRi), during this period. The tachograms of RRi were transferred using an infrared interface device to the Polar Precision Performance SW software v. 3.0 (Polar, Finland), which automatically corrects RRi based on moving average filter. All records of sample data showed were then saved to ".txt" files.

In a second visit $48 \mathrm{~h}$ after the first one, a maximal cardiopulmonary exercise test was performed using a mechanically braked cycle ergometer 167 (ERGOFIT, Germany). The aerobic fitness of the participants was expressed by the values of $\mathrm{VO}_{2}$ max at peak conditions. For safety concerns, electrocardiogram was monitored during the whole test using a multiparametric monitor (Dixtal, Brazil).

The protocol was divided in three phases: 4 min at rest in sitting position; incremental workload until exhaustion $(25 \mathrm{~W} / \mathrm{min}$, maintaining 50 to $60 \mathrm{rpm}$ ); and a $15 \mathrm{~min}$ recovery, the first $3 \mathrm{~min}$ consisting of active resting (cycling to $12.5 \mathrm{~W}$ ), followed by a final 12 min passive rest period. Throughout the tests, the pulmonary gas exchange variables were determined breath-by-breath withVO metabolic analyser (MedGraphics, EUA) calibrated in automatic mode before each test. 
The oxygen consumption and other variables were continuously drawn from facemask connection through sample intervals of 20 s.

\section{Heart Rate Variability Analysis}

All signal analysis were performed with programs in Matlab version 6.5 (The MathWorks, USA) and HRV analysis was performed by the SinusCor Matlab package software (Pulmonary Engineering, PEB/COPPE/UFRJ, Brazil).

The classical HRV analysis was performed in the time domain parameters: reflect the mean percentage of times in which the change in consecutive normal sinus (NN) intervals exceeds 50 milliseconds (pNN50), the standard deviation of all $\mathrm{NN}$ intervals (SDNN) and the root mean square of SDs between adjacent $\mathrm{N}-\mathrm{N}$ intervals (RMSSD). The spectral analysis of the sequence of $\mathrm{RRi}$ was interpolated by cubic splines and resampled with a frequency of $4 \mathrm{~Hz}$ to obtain equally sampled signal and, posteriorly, the spectral parameters were analysed by Welch Periodogram Method (Task Force, 1996) (segments of 256 points with 128 points of overlap using Hanning window): lowfrequency (LF; 0.04-0.15 Hz), high-frequency (HF; $0.15-0.40 \mathrm{~Hz}$ ) and ratio LF/HF. All these parameters were computed in both groups as recommended by the Task Force of the European Society of Cardiology and the North American Society of Pacing and Electrophysiology (Task Force, 1996). All signal analysis was performed with programs written in Matlab version 6.5 (The MathWorks, USA).

\section{Phase-rectification of RR-interval series}

Following the original proposal of Bauer et al. (2009), the decreasing and increasing phases of heart rate were also analysed separately to better estimate the contributions of parasympathetic and sympathetic control, respectively. In the present study, a simplification was proposed. Firstly, it was created a vector of the differences between successive elements of the RRi series; then, the cardiac-deceleration rate (CDR) index was defined as the mean of the positive values, and the cardiac acceleration rate (CAR) index as the mean of the negative values.

\section{Statistical Analysis}

Descriptive statistical analyses of the data were expressed as mean \pm standard deviation. The Kolmogorov-Smirnov test confirmed the normality of distribution. The difference between groups in anthropometric and physical characteristics, the resting heart rate, the $\mathrm{VO}_{2} \mathrm{max}$, and the HRV parameters including CDR and CAR indexes were tested using an independent sample $\mathrm{T}$ test. The model for predicting the $\mathrm{VO}_{2}$ max values through the logistic distribution function are probabilities where 0 corresponds to HG and 1 to LG. A multivariate logistic regression (stepwise) was applied, assuming as independent variables the HRV parameters, including the CDR and CAR indices. The adjustment was selected by the least squared error, and the Wald test results were examined for individual parameters (Hosmer \& Lemeshow, 2000).

Thus, it was obtained a confusion matrix for plotting ROC curve with better sensitivity, specificity and global accuracy, and the area under the curve (AUC) with standard error was used for expressing the overall discriminatory power with $95 \%$ confidence interval $(95 \% \mathrm{CI})$ (Hanley \& Mcneil, 1983). All procedures assumed $\mathrm{p} \leq 0.05$ for statistical significance and were processed in the SPSS 18.0 software (SPSS, USA).

\section{RESULTS}

Anthropometric and physical characteristic of the participants were very similar as well as the low values of standard deviation for each variable (Table 1), showing no significant differences. The $\mathrm{VO}_{2} \max$ of participants with high aerobic fitness was significantly higher than those from participants with low aerobic fitness $\left(48.61 \pm 6.70 \quad\right.$ vs $\quad 34.62 \pm 3.81 \mathrm{ml} \cdot \mathrm{kg}^{-1} \cdot \mathrm{min}^{-1}$, respectively), which made it possible to divide into two groups HG and LG. Besides, the resting heart rate was lower in HG $(53.00 \pm 6.54$ vs $62.71 \pm 6.13, \mathrm{p}=0.001)$ than in LG.

Afterwards, the HRV parameters including CDR and CAR indexes were estimated in both groups and respective inter-group comparison are showed in Table 2. The HG showed significant higher values in all time domain parameters, as well as in the high frequency 
(HF) contents in the frequency domain, compared with LG. Besides, the CDR index of
HG participants was significantly higher than those from LG $(p=0.001)$.

Table 1

Physical and anthropometric characteristics of each group

\begin{tabular}{cccc}
\hline Variables & HG & LG & P-value \\
\hline Age (years) & $21.93 \pm 2.82$ & $22.62 \pm 2.53$ & 0.28 \\
\hline Height (cm) & $175.70 \pm 5.47$ & $174.82 \pm 6.21$ & 0.54 \\
\hline Body Mass (kg) & $71.42 \pm 8.2$ & $73.23 \pm 8.47$ & 0.17 \\
\hline Body Fat Percentage (\%) & $11.54 \pm 2.2$ & $9.82 \pm 2.01$ & 0.07 \\
\hline Resting Heart Rate (bpm) & $53.00 \pm 6.54$ & $62.71 \pm 6.13$ & $0.001^{*}$ \\
\hline $\mathrm{VO}_{2}$ max (ml.kg $\left.{ }^{-1} \mathrm{~min}^{-1}\right)$ & $48.61 \pm 6.70$ & $34.62 \pm 3.81$ & $0.001^{*}$ \\
\hline
\end{tabular}

Values are mean \pm standard deviation (SD); ${ }^{*}$ Significant difference in t-test

Table 2

Comparison of hate rate variability parameters in the time and frequency domains at rest of each group

\begin{tabular}{cccccc}
\hline HRV Variables & HG & $95 \%$ CI & LG & $95 \%$ CI & P-value \\
\hline RMSSD $(\mathrm{ms})$ & $74.21 \pm 28.70$ & 66.07 to 82.70 & $58.72 \pm 24.74$ & 50.27 to 66.45 & $0.01^{*}$ \\
\hline SDNN $(\mathrm{ms})$ & $83.02 \pm 25.23$ & 73.52 to 93.28 & $69.21 \pm 23.35$ & 60.73 to 78.48 & $0.02^{*}$ \\
\hline pNN50 $(\%)$ & $42.64 \pm 18.54$ & 36.01 to 48.35 & $31.38 \pm 17.44$ & 24.29 to 38.78 & $0.01^{*}$ \\
\hline LF $\left(\mathrm{ms}^{2} / \mathrm{Hz}\right)$ & $1812.86 \pm 1481.32$ & 1409.54 to 2268.05 & $1802.73 \pm 1562.88$ & 1412.25 to 2204.60 & 0.97 \\
\hline HF $\left(\mathrm{ms}^{2} / \mathrm{Hz}\right)$ & $2206.86 \pm 1481.37$ & 1709.54 to 2724.41 & $1420.25 \pm 1084.51$ & 1009.70 to 1827.83 & $0.01^{*}$ \\
\hline LF/HF & $1.08 \pm 0.77$ & 0.82 to 1.33 & $1.52 \pm 1.37$ & 1.10 to 1.96 & 0.09 \\
\hline CDR index $(\mathrm{ms})$ & $77.1 \pm 25.72$ & 72.60 to 82.16 & $36.8 \pm 14.01$ & 27.29 to 42.78 & $0.001^{*}$ \\
\hline CAR index $(\mathrm{ms})$ & $-44.81 \pm 17.32$ & -39.54 to 49.19 & $-50.25 \pm 25.57$ & -46.32 to 54.50 & 0.34 \\
\hline
\end{tabular}

Values are mean \pm standard deviation (SD) and $95 \%$ CI is $95 \%$ confidence interval; ${ }^{*}$ Significant difference in t-test

The stepwise logistic regression produced a model including only two variables: CDR index and pNN50. The model showed $85.74 \%$ sensitivity, $88.51 \%$ specificity and $87.16 \%$ global accuracy with standard errors of $0.02(\mathrm{p}=0.04)$ and the AUC was obtained in 0.95 (0.87 to 0.99 with $95 \% \mathrm{CI}$ ), according to equation 1 for predicting probability of the $\mathrm{VO}_{2} \max$ $\left(\mathrm{PVO}_{2} \mathrm{max}\right)$ :

$$
P \dot{V} O_{2 \max }=\frac{e^{-9.7+0.15^{*} C D R+0.05^{*} p N N 50}}{1+e^{-9.7+0.15^{*} C D R+0.05^{*} p N N 50}}
$$

where CDR is cardiac-deceleration rate index and pNN50 is the mean percentage of times in which the change in consecutive normal sinus intervals exceeds $50 \mathrm{~ms}$.

The Odds ratio were calculated by the coefficients presented in the equation are showed in Table 3.

Table 3

Odds ratio calculated by the coefficients of the model

\begin{tabular}{cccc}
\hline Variables & Coefficients & Odds Ratio & $95 \%$ CI \\
\hline CDR index & 0.15 & 1.16 & 1.07 to 1.24 \\
\hline pNN50 & 0.05 & 1.04 & 1.00 to 1.09 \\
\hline Constant & -9.7 & - & - \\
\hline
\end{tabular}

\section{DISCUSSION}

This study aimed to identify participants with different maximum oxygen uptake based on resting HRV analysis and the phase-rectified signal averaging method. The logistic regression model was suitably adjusted to maximal aerobic power data from young healthy males, previously dichotomized into two groups. This represented an important step for stratifying the degree of aerobic fitness without the need of a maximum stress test, with potential application to participants who could not be submitted to such exercise.

Previous studies (De Meersman, 1996; Yataco, Fleisher, \& Katzel, 1997) have compared HRV between high and low aerobic fitness participants, and high aerobic fitness subjects presented higher values of HF power and increase in time domain parameters of the HRV related to parasympathetic activity. Nevertheless, other studies failed to show a relationship between the degree aerobic fitness and HRV in the time and frequency domains (Grant et al., 2009; Melanson \& Freedson, 2001). 
Current findings indicated that participants in HG showed significant higher values of HF power and time domain HRV parameters than the LG. Although both groups contained healthy participants with HRV parameters in the normal range, HG participants presented significant lower resting heart rate. Previous studies have been conducted regarding the mechanisms responsible for the resting bradycardia in high aerobic fitness participants (Buchheit et al., 2010; Plews et al., 2013; Sullo et al., 2003). Changes in the intrinsic mechanisms acting on the sinus node and changes in the autonomic nervous system control of the heart have been reported to contribute to chronic effect of cardiorespiratory training (Carter, Banister, \& Blaber, 2003). These findings lead to the hypothesis that high aerobic conditioning is related to high cardiac efficiency, with improved ejection fraction. In this condition, lower heart rates are required to maintain the arterial blood pressure, and this is obtained by increased vagal tonus. To better investigate this hypothesis, the phase rectified HRV analysis was performed.

In the original PRSA method proposed by Bauer et al. (2009), each positive change of R-R time series was used as the anchor for the coherent average of the surrounding R-R interval segment, and the resulting step was defined as the decelerating capacity index. Similarly, the accelerating capacity index corresponded to the respective step obtained from the negative R-R changes. Furtherly, Nasario-Junior et al. $(2014 ; 2015 a)$ proposed a change to this method, by considering as anchor for the coherent average only the steepest change in each period of positive or negative changes of R-R time series. This approach was successful for stratifying athletes with high aerobic fitness from normal participants. In the present study, the proposal was to simplify the method by avoiding the calculus of coherent averages, since the only interest was on the height of the resulting step. Thus, the mean value of positive changes was taken as a cardiac deceleration rate and the respective value of negative changes as a cardiac acceleration rate.

As the HG participants presented lower resting heart rate when compared to LG, it was supposed they were susceptible to higher vagal tonus, and thus able to present higher values of CDR than LG, as observed. The absence of differences in CAR also supports this hypothesis, since both groups had similar sympathetic tonus at rest.

The result of the logistic regression model supported the hypothesis that CDR index adequately represents the parasympathetic control of the heart rate, and thus it could be applicable to estimate the positive adaptation to cardiorespiratory fitness (Buchheit et al., 2010; Bucheit \& Ginder, 2006; De Meersman, 1996; Martinelli et al., 2005; Moreira et al., 2013; Plews et al., 2013; Yataco, Fleisher, \& Katzel, 1997), or the autonomic control of post-exercise heart rate (Martinelli et al., 2005; Moreira et al., 2013; Plews et al., 2013) and the increasing vagal activity (Trevizani, Belchimol-Barbosa, \& Nadal, 2012). Therefore, it is possible to speculate that regular aerobic exercise, with enough intensity to cause further increase in $\mathrm{VO}_{2}$ max could be evaluated by the CDR index. This variable express changes in heart rate due to parasympathetic control (Buchheit et al., 2010; Bucheit \& Ginder, 2006; Moreira et al., 2013), in agreement with the higher chance showed by CDR index through odds ratio analysis. However, CAR index did not present statistical differences because this is related to the sympathetic control of the heart rate, and not associated with physical fitness (Bauer et al., 2006; Nasario-Junior et al., 2015a). Additionally, the pNN50 is also associated with increased parasympathetic modulation, being directly related to aerobic fitness (De Meersman, 1996; Yataco, Fleisher \& Katzel, 1997).

To the extent of our knowledge, the presented model is the first to stratify the aerobic fitness from parameters derived only from heart rate variability at rest. It was applied with a homogeneous sample of young (18-27 years) health adult male participants, physically active college students, but not athletes, since presented a limited range of $\mathrm{VO}_{2}$ maxvalues, with levels of aerobic fitness $\left(41.6 \pm 8.2 \mathrm{ml} . \mathrm{kg}^{-}\right.$ $\left.{ }^{1} \cdot \mathrm{min}^{-1}\right)$ near the population mean for young people $\left(40 \mathrm{ml} \cdot \mathrm{kg}^{-1} \cdot \mathrm{min}^{-1}\right.$, according to the American College of Sports Medicine) (ACSM, 
2010). In fact, the sample characteristics could be viewed as a limitation, and the potential application to other samples, including both genders and participants who could not be submitted to a maximal cardiopulmonary test is still to be investigated.

\section{CONCLUSION}

The use of the proposed CDR index with the pNN50 parameter measured at rest seems to be able to stratify the level of aerobic fitness in young healthy adult males. Thus, this is performed without the need of applying a stress test and extends the HRV applicability in the evaluation of the physical performance.

\section{Acknowledgments}

This work was partially supported by the Brazilian Research Council (CNPq) and CAPES Foundation.

\section{Conflict of interests:}

Nothing to declare.

Funding:

Nothing to declare.

\section{REFERENCES}

American College of Sport Medicine. (2010). ACSM's Guidelines for Exercise Testing and Prescription. (W. R. Thompson, N. F. Gordon, \& L. S. Pescatello, Eds.) ( $8^{\text {th }}$ Ed.). Philadelphia: Lippincott Williams \& Wilkins.

Bauer, A., Kantelhardt, J. W., Barthel, P., Schneider, R., Mäkikallio, T., Ulm, K., ... Schmidt, G. (2006). Deceleration capacity of heart rate as a predictor of mortality after myocardial infarction: cohort study. Lancet, 20(367), 167481. doi:10.1016/S0140-6736(06)68735-7

Bosquet, L., Gamelin, F. X., \& Berthoin, S. (2007). Is aerobic endurance a determinant of cardiac autonomic regulation? European Journal of Applied Physiology, 100(3), 363-9. doi:10.1007/s00421007-0438-3

Buchheit, M., \& Gindre, C. (2006). Cardiac parasympathetic regulation: respective associations with cardiorespiratory fitness and training load. American journal of physiology. Heart and Circulatory Physiology, 291 (1), H451-458. doi: 10.1152/ajpheart.00008.2006

Buchheit, M., Chivot, A., Parouty, J., Mercier, D., Al Haddad, H., Laursen, P. B., \& Ahmaidi, S. (2010). Monitoring endurance running performance using cardiac parasympathetic function. European Journal of Applied Physiology, 108(6), 1153-1167. doi: 10.1007/s00421-0091317-x

Carnevali, L., \& Sgoifo, A. (2014). Vagal modulation of resting heart rate in rats: the role of stress, psychosocial factors, and physical exercise. Frontiers in Physiology, 5, 1-12. doi: 10.3389/fphys.2014.00118

Carter, J. B., Banister, E. W., \& Blaber, A. P. (2003). Effect of endurance exercise on autonomic control of heart rate. Sports Medicine, 33(1), 3346.

De Meersman, R. E. (1993). Heart rate variability and aerobic fitness. American Heart Journal, 125(3), 726-731.

Grant, C. C., Clark, J. R., Janse van Rensburg, D. C., \& Viljoen, M. (2009). Relationship between exercise capacity and heart rate variability: supine and in response to an orthostatic stressor. Autonomic Neuroscience, 151 (2), 186-188. doi: 10.1016/j.autneu.2009.08.015

Grant, C. C., Murray, C., Janse Van Rensburg, D. C., Fletcher, L. (2013). A comparison between heart rate and heart rate variability as indicators of cardiac health and fitness, Frontiers in Physiology, 4(337), 1-5. doi: 10.3389/fphys.2013.00337

Hanley, J. A., \& Mcneil, B. J. (1983). A method of comparing the areas under receiver operating characteristic curves derived from the same cases. Radiology, 148(3), 839-43.

Hosmer, D. W., \& Lemeshow, S. (2000). Applied Logistic Regression. New York: Wiley.

Jackson, A. S., \& Pollock, M. (1978). Generalized equations for predicting body density of men. The British Journal of Nutrition, 40(3), 497-504.

Kawaguchi, L. Y. A., Nascimento, A. C. P., Lima, M. S., Frigo, L., Júnior, P., De, A. R., ... LopesMartins, R. A. B. (2007). Characterization of heart rate variability and baroreflex sensitivity in sedentary individuals and male athletes. Revista Brasileira de Medicina Do Esporte, 13(4), 231-236. https://doi.org/10.1590/S151786922007000400004

Leite A., Silva, M. E., \& Rocha, A. P. (2013). Heart rate variability analysis in healthy subjects, patients suffering from congestive heart failure and heart transplanted patients. Motricidade, 9(4),54-63. doi: 10.6063/motricidade.9(4).1139

Maroco, M., Nadal, J., \& Barbosa, P. R. (2007). The effect of an aerobic training program on the electrical remodelling of the heart: Highfrequency components of the signal-averaged electrocardiogram are predictors of the maximal aerobic power. Brazilian Journal of Medical and Biological Research, 40(2), 199-208. doi: 10.1590/S0100-879X2006005000068

Martinelli, F. S., Chacon-Mikahil, M. P. T., Martins, L. E. B., Lima-Filho, E. C., Golfetti, R., Paschoal, M. A., \& Gallo-Junior, L. (2005). Heart rate variability in athletes and non-athletes at rest and during head-up tilt. Brazilian Journal of 
Medical and Biological Research, 38(4), 639-647. doi: 10.1590/S0100-879X2005000400019

Melanson, E. L., \& Freedson, P. S. (2001). The effect of endurance training on resting heart rate variability in sedentary adult males. European Journal of Applied Physiology, 85(5), 442-449. doi: $10.1007 / \mathrm{s} 004210100479$

Mezzani, A., Agostini, P., Cohen-Solal, A., Corrà, U., Jegier, A., Kouidi, E., ... Vanhees, L. (2009). Standards for use of cardiopulmonary exercise testing for the functional evaluation of cardiac patients: a report from the Exercise Physiology Section of the European Association for Cardiovascular Prevention and Rehabilitation. European Association of Cardiovascular Prevention and Rehabilitation, 16(3), 249-267. doi: 10.1097/HJR.0b013e32832914c8

Moreira, D. N., Peçanha, T., Guerra, Z. F., Silva, L. P., Nakamura, F. Y., Laterza, M. C., \& de Lima, J. R. P. (2013). Effects of aerobic fitness on cardiac autonomic modulation during supine and upright posture. Journal of Exercise Physiology Online, 16(4), 92-100.

Nasario-Junior, O., Benchimol-Barbosa, P. R., Pedrosa, R. C., \& Nadal, J. (2014). Refining the deceleration capacity index in phase-rectified signal averaging to assess physical conditioning level. Journal of Electrocardiology, 47(3), 306-310. doi: 10.1016/j.jelectrocard.2013.12.006

Nasario-Junior, O., Benchimol-Barbosa, P. R., Pedrosa, R. C., \& Nadal, J. (2015a). Beat-to-beat ventricular repolarization duration variability assessed by cardiac acceleration and deceleration phases in athletes. EC Cardiology, 1, 33-42.

Nasario-Junior, O., Benchimol-Barbosa, P. R., Pedrosa, R. C., \& Nadal, J. (2015b). Assessment of autonomic function by phase rectification of RR-Interval histogram analysis in Chagas disease. Arquivos Brasileiros de Cardiologia, 104(6), 450-456. doi: 10.5935/abc.20150032

Peçanha, T., de Paula-Ribeiro, M., Nasario-Junior, O., \& de Lima, J.R. (2013). Post-exercise heart rate variability recovery: a time-frequency analysis.
Acta Cardiologica, 68(6), 607-613. doi: 10.2143/AC.68.6.8000008

Plews, D. J., Laursen, P. B., Stanley, J., Kilding, A. E., \& Bucheit, M. (2013). Training adaptation and heart rate variability in elite endurance athletes: opening the door to effective monitoring. Sports Medicine, 43(9), 773-781. doi: 10.1007/s40279-013-0071-8

Rodríguez, J. D., Pérez, A., \& Lozano, J. A. (2010). Sensitivity analysis of K-fold Cross validation in prediction error estimation. IEEE transactions on pattern analysis and machine intelligence, 32(3), 569575. doi: 10.1109/TPAMI.2009.187

Rosenwinkel, E. T., Bloomfield, D. M., Arwady, M. A., \& Goldsmith, R. L. (2001). Exercise and autonomic function in health and cardiovascular disease. Cardiology Clinics, 19(3), 369-87.

Siri, W. E. (1961). Body composition from fluid spaces and density: analysis of methods. In J. Brozek \& A. Henschel (Eds.), Techniques for measuring body composition. Washington: National Academy of Science.

Task Force of the European Society of Cardiology and the North American Society of Pacing and Electrophysiology. (1996). Heart rate variability: standards of measurement, physiological interpretation and clinical use. Circulation Research, 93, 1043-1065. doi:10.1161/01.CIR. 93.5.1043

Trevizani, G. A., Belchimol-Barbosa, P. R., \& Nadal, J. (2012). Effects of age and aerobic fitness on heart rate recovery in adult men. Arquivos Brasileiros de Cardiologia, 99(3), 802-810. doi: 10.1590/S0066-782X2012005000069

Wasserman, K., \& Whipp, B. J. (1975). Exercise physiology in health and disease. The American Review of Respiratory Disease, 112, 219-249. doi:10.1164/arrd.1975.112.2.219

Yataco, A. R., Fleisher, L. A., \& Katzel, L. I. (1997). Heart rate variability and cardiovascular fitness in senior athletes. American College of Cardiology, 80(10), 1389-1391. doi:10.1016/S00029149(97)00697-8 